\title{
Reconciling change blindness with long-term memory for objects
}

\author{
${\text { Katherine } \text { Wood }^{1} \text { (D) - Daniel J. Simons }}^{1}$
}

Published online: 23 November 2016

(C) The Psychonomic Society, Inc. 2016

\begin{abstract}
How can we reconcile remarkably precise longterm memory for thousands of images with failures to detect changes to similar images? We explored whether people can use detailed, long-term memory to improve change detection performance. Subjects studied a set of images of objects and then performed recognition and change detection tasks with those images. Recognition memory performance exceeded change detection performance, even when a single familiar object in the postchange display consistently indicated the change location. In fact, participants were no better when a familiar object predicted the change location than when the displays consisted of unfamiliar objects. When given an explicit strategy to search for a familiar object as a way to improve performance on the change detection task, they performed no better than in a 6-alternative recognition memory task. Subjects only benefited from the presence of familiar objects in the change detection task when they had more time to view the prechange array before it switched. Once the cost to using the change detection information decreased, subjects made use of it in conjunction with memory to boost performance on the familiar-item change detection task. This suggests that even useful information will go unused if it is sufficiently difficult to extract.
\end{abstract}

Keywords Memory:Long-termmemory · Changeblindness · Object Recognition

Katherine Wood

kmwood2@illinois.edu

1 Department of Psychology, University of Illinois, 603 E. Daniel Street, Champaign, IL 61820, USA
Images viewed for only a moment can persist in memory. People recognize photographs they had viewed for just seconds with 98\% accuracy after several hours (Shepard, 1967) and with $63 \%$ accuracy after a year (Nickerson, 1968). They remain highly accurate even after viewing thousands of images in a single sitting (Standing, 1973; Standing, Conezio, \& Haber, 1970). This apparently enormous-capacity, long-term visual memory is highly detailed. After spending 5.5 hours in a single day viewing 2,500 images of objects for $3 \mathrm{~s}$ each, subjects could distinguish objects they had studied from highly similar ones (I saw this balloon, not that balloon) and from the same objects in different states (I saw the open breadbox, not the closed one) with approximately $90 \%$ accuracy (Brady, Konkle, Alvarez, \& Oliva, 2008).

Despite having detailed, long-term recognition memory for objects and scenes, people fail to notice changes from one object to another when the change occurs during a fractionof-a-second delay. This change blindness occurs even for differences much larger than those that observers can correctly discriminate in long-term recognition memory; many people fail to notice when the person to whom they are giving directions is replaced by someone else (Simons \& Levin, 1998), when two people in a picture switch heads (Grimes, 1996), and when objects disappear or move (Rensink, O'Regan, \& Clark, 1997). Why can we remember whether a breadbox was open or closed after a delay of hours, yet fail to notice a change from one breadbox to another after a delay of milliseconds?

Like long-term memory for objects, change detection is stable across varying delays. Performance in one-shot change detection tasks is similar with delays of $250 \mathrm{~ms}$ and $4 \mathrm{~s}$ (Simons, 1996), and subjects could detect changes to studied images just as well with immediate testing as after a 10-min delay, remaining above chance a day later (Hollingworth, 2005). In other words, people store scene details well enough to detect changes regardless of the 
length of the intervening delay, but fail to use that information when tasked with immediate, moment-to-moment change detection.

Long-term memory can facilitate change detection in some ways, however. People are faster to detect a change when it reoccurs, and they better detect new changes occurring at previously changed locations in a scene (Becker \& Rasmussen, 2008). By guiding attention to previously relevant locations in familiar scenes, remembered details aid change detection for elements within the scene.

Although a familiar scene context aids change detection, no study has systematically explored whether familiarity with the individual objects themselves affects change detection for those objects. When objects are repeated within a change detection task, people are better able to detect identity changes to them (Simons, 1996), but that form of familiarity might just reflect more efficient encoding of objects within the task itself. In four studies, we examine whether highly detailed long-term memory for objects improves change detection performance in displays using those objects. Our study design incorporates a smaller-scale replication of the tasks used to demonstrate exceptional long-term memory for objects (Brady et al., 2008).

\section{General methods}

Our methods, procedures, target sample size, exclusion rules, stimuli, experimental code, and analysis scripts were preregistered prior to data collection. Data were analyzed using R (R Core Team, 2015) with the BayesFactor (Morey \& Rouder, 2015) and bootES (Kirby \& Gerlanc, 2013) libraries, and all scripts and data are also available (https://osf.io/6 y35t/). Both in the manuscript and in our preregistration, we report all data exclusions, all manipulations, and all measures (Simmons, Nelson, \& Simonsohn, 2011).

\section{Materials and equipment}

All tasks were coded in the Psychopy package for Python (Peirce, 2007) and presented using Apple Mac Mini computers on 24-in. BenQ XL2420TX monitors $(1920 \times 1080$ at $100 \mathrm{~Hz}$ ). Subjects sat with their head resting on a custom-built chin rest positioned $55 \mathrm{~cm}$ from the monitor.

Stimuli were objects taken from the Massive Memory database (http://cvcl.mit.edu/MM/), supplemented with similar objects from Google Images. Each image appeared against a white background and was scaled to fit within an $8.5 \times 8.5$ degree white square while preserving its aspect ratio.

From the complete set of 2,767 objects, we created two sets of images. One set consisted of 1,965 items, each from a different category. These unstudied objects served as filler items for recognition and change detection tasks. The remaining 802 items consisted of pairs of tokens from a category, with each pair representing a unique category (two different teddy bears, two different rubber ducks, two toasters, and so on).

In each experiment, subjects first studied a set of objects and then completed a set of recognition memory and change detection tasks. No objects were repeated within or across tasks for a participant, and in each task all of the filler items were unique on each trial. The primary difference between experiments consisted of the particular tasks used and the instructions given to subjects.

\section{Tasks}

Each task is described below, and the sequence of events for each task is depicted schematically in Fig. 1.

Study task Subjects studied a sequence of objects from the set of exemplar pairs. One randomly selected member of each pair served as a studied item and the other served as a lure item for use in later tasks. Each image appeared at fixation for $3 \mathrm{~s}$, followed by a 500-ms interstimulus interval (ISI). At fixed intervals, subjects were given untimed breaks. They initiated the next portion of the study phase with a keypress.

Change detection with a familiar item On each trial, subjects viewed an array of six objects, equally spaced in an annulus with a radius of 12.6 degrees of visual angle. This prechange array appeared for $1,200 \mathrm{~ms}$, followed by a white screen for $400 \mathrm{~ms}$, and then by a postchange array that remained onscreen until subjects responded. Five of the objects in the prechange display were drawn at random, without replacement, from the set of filler objects. The sixth object was the unstudied pair member corresponding to a randomly selected studied exemplar. In the postchange array, the unstudied exemplar always changed into its studied counterpart. Every trial included a change, and subjects responded by clicking the mouse cursor on the changed object. They were asked to guess if they did not detect a change.

Change detection with all unfamiliar items This task was identical to the change detection task with a familiar object except that none of the objects had been studied. Again, the change involved a switch between two members of a category.

6AFC memory task One randomly selected, previously studied object and five randomly selected filler objects were arrayed in an annulus with a 12.6 degree radius. Subjects had unlimited viewing time and were instructed to click on the object they had seen during the study phase.

2AFC memory task A studied exemplar and its unstudied partner from the same category were presented on screen at 


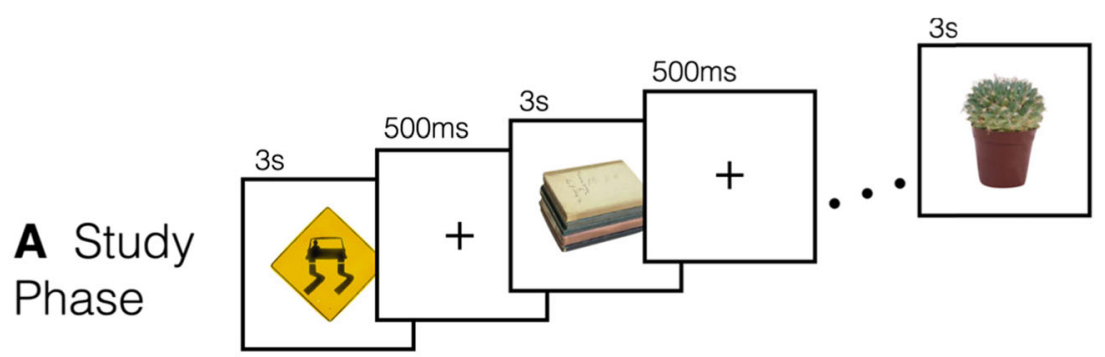

B Change

Detection Unfamiliar Items

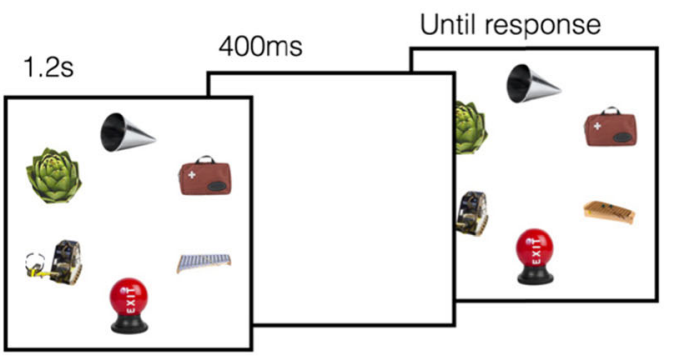

\section{Change Detection - Familiar Items}

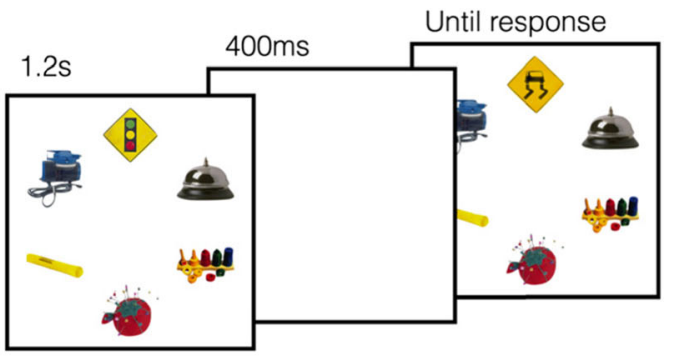

Until response

\section{2AFC Memory Task}

Fig. 1 a Study phase consisting of a series of random, nonrepeating members of exemplar pairs. b Change detection with unfamiliar items. An unstudied exemplar changes into its counterpart (in this case, the xylophone). c Change detection with familiar items. The unstudied half of an exemplar pair changes into its studied counterpart (here, the

15.8 degrees eccentricity. Subjects had unlimited viewing time, and indicated the object they had seen before with the arrow keys (left arrow for the image on the left side of the screen, right arrow for the right side). stoplight sign changes into the studied winding road sign). d Twoalternative, forced-choice memory task. A studied exemplar is paired with its unstudied counterpart. e Six-alternative, forced-choice memory task. A studied exemplar is presented with five randomly selected, nonrepeating filler objects

\section{Experiment 1}

Experiment 1 was designed to examine both long-term memory and change detection performance using a 
common set of objects to explore how performance differs across those tasks and whether long-term memory can affect change detection performance. Our design included a replication of the technique used to demonstrate excellent long-term object memory, albeit with a smaller set of objects (Brady et al., 2008).

\section{Method}

Thirty undergraduate students at the University of Illinois Urbana-Champaign participated in the experiment for course credit. Subjects completed five tasks in the following sequence, with untimed breaks between tasks: first, a study phase (240 images), followed by change detection with a familiar object (80 trials), change detection with only unfamiliar objects (80 trials), a six-alternative recognition memory task ( 80 trials), and finally a two-alternative recognition memory task (80 trials).

\section{Results and discussion}

Prior to analysis, we excluded data from three subjects whose accuracy fell below our preregistered inclusion criteria (20\% on the $6 \mathrm{AFC}$ memory task or $55 \%$ on the $2 \mathrm{AFC}$ memory task), leaving data from 27 subjects in the final analysis. Figure 2 shows the results of all four experiments for all tasks included in each experiment, and Table 1 summarizes the results of all four experiments.

Performance was comparable across the two change detection tasks, with substantial change blindness (see Table 2). Subjects averaged $46 \%$ detection $(S D=14 \%)$ for the familiar object change and $48 \%$ detection $(S D=10 \%)$ for the unfamiliar object change, $t(26)=1.03$ (paired, two-tailed), $p=.32$, Cohen's $d=$ $0.20, \mathrm{BF}_{10}=0.33$. The Bayes factor shows that the data are about 3 times as likely under the null hypothesis as under a default alternative hypothesis based on a two-tailed Cauchy distribution with a median effect size of 0.707 (50\% of the values in the distribution fall between -0.707 and 0.707 ). We elected to use a prior that weights the null and alternative hypotheses as equally likely; this decision is arbitrary and debatable, but allows for a direct comparison of the evidence provided by these Bayes factors. All of our reported Bayes factors use the same prior and default alternative distribution. Cohen's $d$ was calculated using a within-subjects procedure (Lakens, 2013).

Not surprisingly, 2AFC memory performance $(M=78 \%, S D$ $=11 \%)$ exceeded $6 \mathrm{AFC}$ performance $(M=63 \%, S D=20 \%)$, $t(26)=5.37, p<.0001, d=1.34, \mathrm{BF}_{10}=1703.94$. However, $2 \mathrm{AFC}$ memory performance was about $10 \%$ lower than the $88 \%$ observed in studies demonstrating exceptional long-term memory for objects (Brady et al., 2008), even though our task placed much smaller memory demands on subjects, with fewer studied objects and a substantially shorter experiment duration.

Subjects did not spontaneously use long-term familiarity to improve their change detection performance. The familiar object
Fig. 2 The shaded region of each violin plot represents a smoothed kernel density, indicating the distribution of participant scores for that condition. The black dots represent each subject's score on that task, and the black horizontal line on each violin plot represents the mean for all subjects in that task. The columns, from left to right, represent scores on the $2 \mathrm{AFC}$ memory task, the $6 \mathrm{AFC}$ memory task, the change detection task with unfamiliar items, the change detection task with familiar items when subjects were not aware of the usefulness of the presence of a familiar item, and the hybrid task in which subjects were told that familiar items could help with change detection. Plots generated with the ggplot2 package for R (Wickham, 2009)

in the postchange array was always the changed object, but subjects either did not notice this link or failed to use it to enhance performance. Had they done so, they could have treated the task as a $6 \mathrm{AFC}$ task, disregarding the prechange array entirely, yet they performed substantially worse than in the 6AFC task, $t(26)$ $=4.20, p<.001, d=.82, \mathrm{BF}_{10}=105.88$. Moreover, they performed no better when familiarity perfectly predicted the location of the change than when all objects were unfamiliar. Apparently, subjects do not use even strongly reliable cues to enhance change detection (Beck, Angelone, Levin, Peterson, \& Varakin, 2008). In this case, we also have direct evidence from their recognition performance that the familiarity information was available to them, but went unused.

\section{Experiment 2}

Although people do not spontaneously use feature-based cues to the location of a change, they can use such cues once informed of them (Beck et al., 2008). Subjects in Experiment 1 did not use the familiarity of the changed object in the postchange display to enhance performance on the task, but perhaps they would if informed in advance that the familiar object was always the changed one. If informed about the usefulness of recognition memory, subjects should perform better on the change detection task with familiar items compared to change detection with unfamiliar items.

\section{Method}

Twenty-seven undergraduate students at the University of Illinois Urbana-Champaign participated in the experiment for course credit. The materials, stimuli, and procedures were identical to Experiment 1 except for the following changes:

1. In place of the unfamiliar object change detection task, subjects completed two blocks of the familiar object change detection task. For the first block, they were uninformed about the predictability of the change location. For the second block, subjects were told that if a familiar object were present in the second array, that was the object that changed from the first array and they should select that as their response. This meant that subjects could disregard the first array of objects they saw entirely and complete the task using long-term memory information alone. 
Unfamiliar Familiar

2AFC 6AFC CD CD (naive) Hybrid

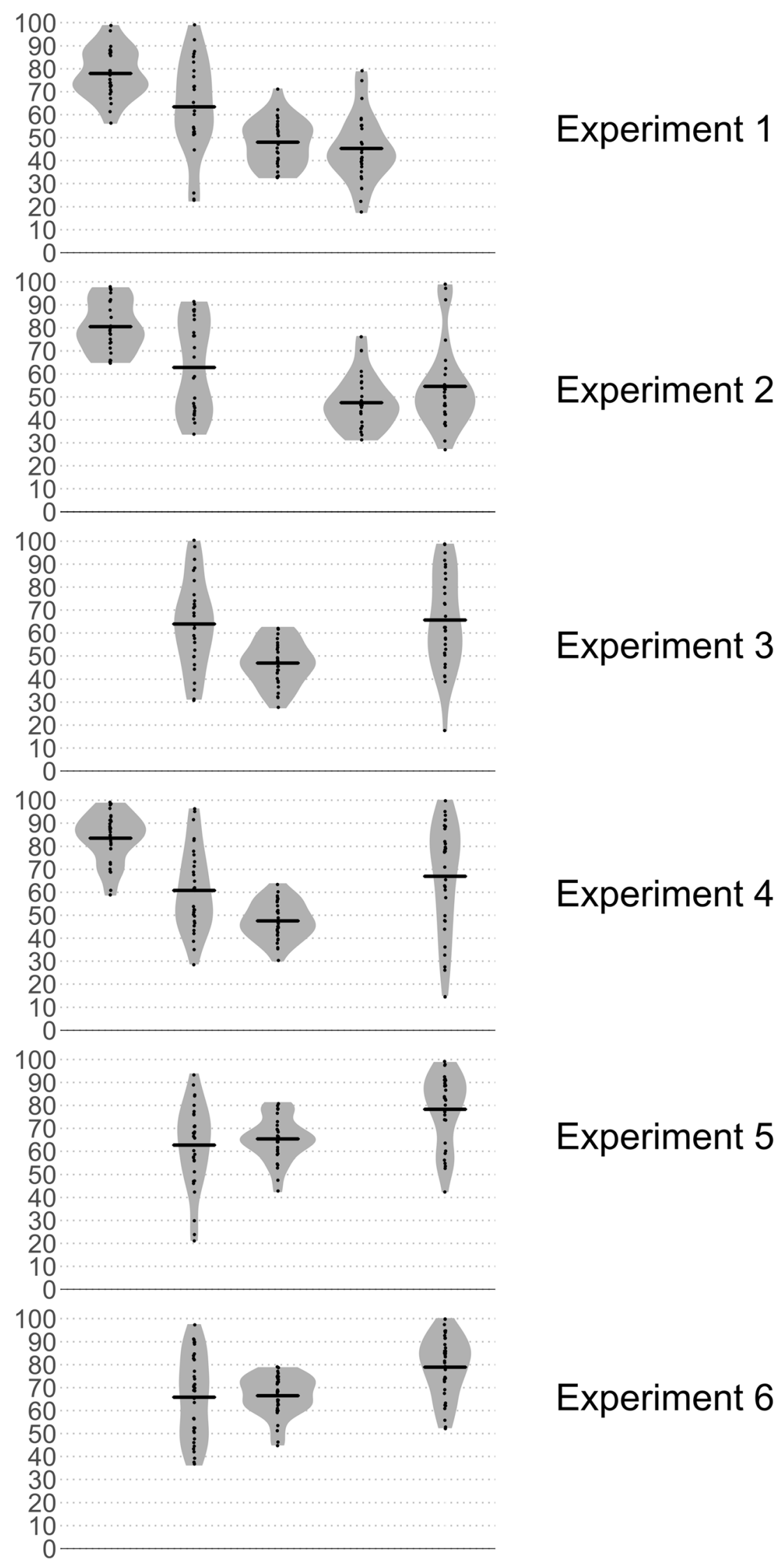


2. The number of studied objects was increased to 320 due to the use of familiar objects in both change detection tasks. Subjects could take an untimed break after studying 107th and 214th images.

\section{Results and discussion}

Prior to analysis, we excluded data from two subjects whose accuracy fell below our preregistered inclusion criteria $(20 \%$ on the 6 AFC memory task or $55 \%$ on the 2 AFC memory test), leaving data from 25 subjects in the final analysis.

Overall, Experiment 2 replicated the results found in Experiment 1, with similar levels of change blindness, similar recognition rates, and $2 \mathrm{AFC}$ recognition performance lower than that reported in earlier long-term memory studies (Brady et al., 2008).

Once again, subjects exhibited a high degree of change blindness both when they were uninformed about the predictiveness of the familiar object $(M=48 \%$ correct, $S D=11 \%)$ and when they were informed $(M=55 \%, S D=19 \%)$. Performance was significantly better for the informed subjects, $t(24)=2.26, p=$ $0.03, d=0.50$, but there was minimal evidence for a difference (relative to the null), with $\mathrm{BF}_{10}=1.76$. Post hoc inspection of the data suggests that the difference was driven by three subjects who achieved greater than $90 \%$ accuracy. Without these subjects, the mean in the informed condition fell to $49 \%(S D=11 \%)$.

Both 6AFC performance $(M=63 \%, S D=20 \%)$ and $2 \mathrm{AFC}$ performance $(M=81 \%, S D=11 \%)$ were comparable to Experiment 1. 6AFC accuracy exceeded levels in both the informed, $t(24)=2.30, p=0.03, d=0.46, \mathrm{BF}_{10}=1.91$, and uninformed, $t(24)=3.76, p<.001, d=.80, \mathrm{BF}_{10}=35.83$, change detection conditions.

With the exception of three high performers, subjects do not appear to have made use of the relationship between the change detection task and the studied items, despite being directly informed of it. Although the presence of a familiar object completely predicted the change and subjects were informed of this fact, accuracy was comparable to a change detection task with completely novel objects. It remains unclear whether the vast majority of subjects did not use the information or could do not use it.

\section{Experiment 3}

Experiment 1 suggested that subjects do not spontaneously use recognition memory to enhance change detection, and Experiment 2 showed that most do not use recognition memory even when informed that it is a perfectly predictive cue. In both experiments, accuracy was no better than for change detection with entirely novel objects. The finding that three subjects in Experiment 2 achieved excellent accuracy raises the possibility that they might have successfully used familiarity information.
Perhaps they adopted a more effective strategy than other subjects for combining information from long-term memory with their search for change. In Experiment 3, we gave subjects a step-by-step algorithm for jointly using long-term memory and change detection to see if such strategic scaffolding would improve accuracy beyond that of either memory or change detection alone.

\section{Method}

Thirty undergraduate students at the University of Illinois Urbana-Champaign participated in the experiment for course credit. This experiment included the familiar- and unfamiliarobject change detection tasks and the 6AFC task, but not the 2AFC task. Given that only two tasks included studied objects, the study phase included 160 images with an untimed break after 80 images. Subjects completed the change detection task with unfamiliar objects, followed by the change detection with familiar objects, and then by the 6AFC task. For the familiar-object change detection trials, subjects were given the following strategy: first, attempt to find the change between the two arrays; second, if they could not detect the change, look for a familiar object in the second array, and if they saw one, select it as their response. Following these trials, subjects typed a description of their approach to the task and reported whether they used the provided strategy. If subjects applied this strategy perfectly, their performance in the change detection task with familiar objects should exceed that of either the $6 \mathrm{AFC}$ or the unfamiliar object change detection task.

\section{Results and discussion}

Prior to analysis, data for one subject were excluded for accuracy levels below the preregistered thresholds, leaving data from 29 subjects in the final analysis.

Change detection performance with unfamiliar objects was comparable to Experiment $1(M=47 \%, S D=10 \%)$ and substantially worse than when were given an explicit strategy to use recognition memory $(M=66 \%, S D=21 \%), t(28)=5.23$, $p<.0001, d=1.11, \mathrm{BF}_{10}=1,465.14$. With this strategy, their change detection performance did not differ significantly from their 6AFC performance $(M=64 \%, S D=19 \%), t(28)=0.59$, $p=.56, d=0.11, \mathrm{BF}_{10}=0.23$. $6 \mathrm{AFC}$ performance was comparable to that of Experiments 1 and 2.

Assuming that the mechanisms and representations underlying short-term change detection and long-term memory are distinct, subjects should have performed better in the familiar item change detection task than in either the unfamiliar item change detection task or the 6AFC task. However, their accuracy rose only to the levels of their 6AFC memory scores; subjects might be able to use one or the other source of information to perform the task but not to combine both sources of information. Alternatively, if both tasks share a representation, 
Table 1 All $t$ tests are paired, two-tailed tests. The Bayes factor shows the likelihood of the data under the null hypothesis compared to a twotailed default alternative hypothesis structured as a Cauchy distribution with a scale of .707 . Cohen's $d_{r m}$ was calculated using a repeated- measures procedure for a within-subjects design to correct for correlations between measures (Lakens, 2013), with 95\% confidence intervals bootstrapped with 2,000 repetitions. The correlation column shows the Pearson correlation between the measures tested

\begin{tabular}{|c|c|c|c|c|c|c|}
\hline Experiment & Comparison & $t$ test & $\mathrm{BF}_{10}$ & Cohen's $d_{r m}$ & $95 \% \mathrm{CI}$ on $d$ & $r$ \\
\hline 1 & Familiar vs. unfamiliar & $t(26)=1.03, p=.32$ & 0.33 & 0.20 & {$[-0.12,0.54]$} & .40 \\
\hline 1 & Familiar vs. $6 \mathrm{AFC}$ & $t(26)=4.20, p<.001$ & 105.88 & 0.82 & {$[0.56,1.19]$} & .19 \\
\hline 1 & $2 \mathrm{AFC}$ vs. $6 \mathrm{AFC}$ & $t(26)=5.37, p<.001$ & $1,703.94$ & 1.34 & {$[1.00,1.93]$} & .75 \\
\hline 2 & Familiar vs. informed & $t(24)=2.26, p=.03$ & 1.76 & 0.50 & {$[0.21,0.82]$} & .55 \\
\hline 2 & Familiar vs. 6AFC & $t(24)=3.76, p<.001$ & 35.83 & 0.80 & {$[0.51,1.22]$} & .24 \\
\hline 2 & Informed vs. $6 \mathrm{AFC}$ & $t(24)=2.30, p=.03$ & 1.91 & 0.46 & {$[0.20,0.76]$} & .58 \\
\hline 3 & Strategy vs. unfamiliar & $t(28)=5.23, p<.001$ & $1,465.14$ & 1.11 & {$[0.82,1.53]$} & .37 \\
\hline 3 & Strategy vs. 6AFC & $t(28)=0.59, p=.56$ & 0.23 & 0.11 & {$[-0.19,0.41]$} & .69 \\
\hline 4 & Strategy vs. unfamiliar & $t(30)=4.48, p<.001$ & 257.02 & 0.90 & {$[0.57,1.50]$} & .06 \\
\hline 4 & Strategy vs. 6AFC & $t(30)=1.49, p=.15$ & 0.52 & 0.27 & {$[-0.002,0.68]$} & .38 \\
\hline 4 & $2 \mathrm{AFC}$ vs. $6 \mathrm{AFC}$ & $t(30)=9.78, p<.001$ & $>100$ mil & 2.03 & {$[1.69,2.60]$} & .68 \\
\hline 5 & Unfamiliar vs. $6 \mathrm{AFC}$ & $t(30)=0.84, p=.41$ & 0.26 & 0.16 & {$[-0.16,0.45]$} & .26 \\
\hline 5 & Unfamiliar vs. informed & $t(30)=5.27, p<.001$ & $1,959.76$ & 1.03 & {$[0.73,1.53]$} & .49 \\
\hline 5 & 6AFC vs. informed & $t(30)=7.20, p<.001$ & $276,145.90$ & 1.32 & {$[1.05,1.84]$} & .75 \\
\hline 6 & Unfamiliar vs. $6 \mathrm{AFC}$ & $t(35)=0.22, p=.83$ & 0.18 & 0.04 & {$[-0.25,0.33]$} & .26 \\
\hline 6 & Unfamiliar vs. informed & $t(35)=6.74, p<.001$ & $176,266.20$ & 1.20 & {$[0.95,1.55]$} & .53 \\
\hline 6 & $6 \mathrm{AFC}$ vs. informed & $t(35)=6.78, p<.001$ & $196,011.20$ & 1.24 & {$[0.99,1.61]$} & .77 \\
\hline
\end{tabular}

it may not be possible to increase performance beyond that of the 6AFC task, which is the easier of the two.

\section{Experiment 4}

In Experiment 4, we again tried to provide subjects with a strategy to boost performance in the change detection task with familiar objects. Perhaps presenting change detectionthe more difficult of the tasks - as the primary task limited their ability to take advantage of both sources of information. In this experiment, we presented recognition of the familiar object as the first step in performing the task, with change detection as a potential aid; the task itself remained the same.

In the first three experiments, the study phase consisted of nothing but viewing the images - subjects had no additional task to complete. All three experiments showed lower recognition performance than expected based on previous reports of exceptional long-term memory for objects (Brady et al., 2008). In those earlier studies, subjects engaged in an $n$-back task during the study phase to ensure that they were attentive. We added an n-back task in Experiment 4 to determine whether our lower recognition rates resulted from reduced engagement during the study phase.

subjects were not aware of the usefulness of the presence of a familiar item, and the hybrid task in which subjects were told that familiar items could help with change detection 2 Means (standard deviations) for performance on each task in 2AFC memory task, the 6AFC memory task, the change detection task with unfamiliar items, the change detection task with familiar items when

\begin{tabular}{|c|c|c|c|c|c|}
\hline \multirow[t]{2}{*}{ Experiment } & \multicolumn{5}{|l|}{ Mean $(S D)$} \\
\hline & $2 \mathrm{AFC}$ & $6 \mathrm{AFC}$ & Unfamiliar CD & Familiar CD (naïve) & Hybrid \\
\hline 1 & $78 \%(11 \%)$ & $63 \%(20 \%)$ & $48 \%(10 \%)$ & $46 \%(14 \%)$ & NA \\
\hline 2 & $81 \%(11 \%)$ & $63 \%(20 \%)$ & NA & $48 \%(11 \%)$ & $55 \%(19 \%)$ \\
\hline 3 & NA & $64 \%(19 \%)$ & $47 \%(10 \%)$ & NA & $66 \%(21 \%)$ \\
\hline 4 & $84 \%(11 \%)$ & $61 \%(18 \%)$ & $48 \%(8 \%)$ & NA & $67 \%(23 \%)$ \\
\hline 5 & NA & $63 \%(18 \%)$ & $65 \%(9 \%)$ & NA & $78 \%(16 \%)$ \\
\hline 6 & NA & $66 \%(18 \%)$ & $67 \%(9 \%)$ & NA & $78 \%(13 \%)$ \\
\hline
\end{tabular}




\section{Method}

Thirty-one undergraduate students at the University of Illinois Urbana-Champaign participated in the experiment for course credit. During the study phase, subjects viewed 240 images, with an untimed break halfway through. In each half of the study phase, three filler objects, unused in any other part of the experiment, were repeated after intervals of 10,30 , and 60 intervening objects, for a total of six pairs of repeated objects. The order of the repetition intervals was randomized for each subject. Subjects were instructed to press the spacebar if they saw a repeated object, and the image turned green to indicate a correctly identified repeat.

Following the study phase, subjects completed the familiar object change detection task followed by the unfamiliar object change detection task, the 6AFC task, and the 2AFC task. For the change detection task with familiar objects, subjects were instructed to first look for a familiar item in the second array, and if they could not find it, to determine which object changed from the first array. After subjects responded by clicking on the familiar object, they were asked to respond with the arrow keys (left for no, right for yes) whether the object they selected had changed from the first array.

\section{Results and discussion}

All 31 subjects surpassed the preregistered accuracy thresholds. Change detection performance for unfamiliar arrays of objects was similar to that of Experiments 1 and $2(M=48 \%$, $S D=8 \%)$. $2 \mathrm{AFC}$ performance $(M=84 \%, S D=11 \%)$ again was significantly better than $6 \mathrm{AFC}$ performance $(M=61 \%$, $S D=18 \%), t(30)=9.78, p<.001, d=2.03, \mathrm{BF}_{10}=1.30 \times 10^{8}$. For the familiar change detection task in which subjects were given an explicit strategy to follow, accuracy $(M=67 \%, S D=$ $23 \%$ ) was better than for change detection with unfamiliar objects, $t(30)=4.48, p<.001, d=0.90, \mathrm{BF}_{10}=257.02$, but comparable to performance in the 6AFC task, $t(30)=1.49, p=$ $0.15, d=0.27, \mathrm{BF}_{10}=0.52$.

Providing a strategy that emphasized long-term memory ahead of change detection did not result in better performance than the opposite emphasis did in Experiment 3. In both cases, accuracy on the change detection task with familiar items mirrored that of the 6AFC memory task. Providing a strategy did not enable them to enhance performance beyond that of recognition memory alone, either because they could not jointly use long-term memory and change detection information, or because their 6AFC performance represents a ceiling that can be reached but not surpassed.

Subjects correctly identified repeats during the study phase $84 \%$ of the time $(S D=15 \%)$, aligning closely with the $80 \%$ accuracy rate found by Brady et al. (2008). Despite incorporating an $n$-back task into the study phase of the experiment, 2AFC memory scores improved only slightly beyond what we observed in Experiments 1-3, remaining below the excellent performance observed in other studies (Brady et al., 2008). Apparently, lower recognition performance in our experiments cannot be entirely explained by a lack of engagement during the study phase.

\section{Experiment 5}

In Experiments 3 and 4, accuracy on the familiar-item change detection task remained lower than accuracy on the 6AFC memory task, despite the availability of two information sources (long-term memory and short-term changes to the display) and an explicit strategy for how to combine them. Subjects did not use of all of the information available to them, but the reason why remains unclear. If both change detection and recognition draw on a shared pool of resources, then performance on the 6AFC task - the easier of the two-might constitute a performance ceiling that can be met but not exceeded. Alternatively, the tasks may use separate resources, but subjects might be unwilling (or unable) to expend additional resources if the payoff does not justify the expense. If so, then they potentially could perform better than they did in the 6AFC case by using change detection information, but they do not because the change detection task is simply too costly to justify the effort.

In Experiment 5, we tried to improve performance on the change detection task by increasing the prechange duration to 5 seconds. Longer presentations of the prechange array lead to greater accuracy (Brady, Konkle, Oliva, \& Alvarez, 2015). If participants potentially could use change detection information to increase their accuracy beyond either the memory task or unfamiliar-item change detection task alone, then reducing the effort necessary to perform the change detection should lead to higher performance.

Prior experience with each task individually prior to combining them might also explain why participants did not appear to use both forms of information to enhance performance. To address that possibility, we altered the task order such that subjects completed the two component tasks before the familiar-item change detection.

\section{Method}

Thirty-three undergraduate students at the University of Illinois at Urbana-Champaign participated in the experiment for course credit.

As in Experiments 1-3, subjects first studied objects for 3 seconds each. Because only two tasks used studied images, subjects studied 160 objects total. They then completed the change detection task with unfamiliar objects as in earlier experiments, but with an increased display time for the initial array in each trial $(5 \mathrm{~s})$. They next completed the 6AFC memory test, followed by the familiar-item change detection task 
(also with a 5-s prechange display time). For the familiar-item task, subjects were instructed that they would be finding changes as they had earlier in the experiment, but that if they saw a familiar object in the postchange array, then it was the object that had changed. As before, subjects were instructed to guess when they were unsure about the correct answer.

\section{Results and discussion}

Two subjects' accuracy failed to meet the pre-registered thresholds, and their data were excluded from analysis, leaving data from 31 subjects.

Accuracy on the 6AFC memory task was consistent with that of other experiments $(M=63 \%, S D=18 \%)$. Unfamiliar-item change detection performance increased with the longer duration $(M=65 \%, S D=9 \%)$ and did not significantly differ from accuracy on the 6AFC task, $t(30)=0.84, p=.41, d=0.16, \mathrm{BF}_{10}=0.26$. Familiaritem change detection performance $(M=78 \%, S D=$ $16 \%)$ was significantly higher than both $6 \mathrm{AFC}$ performance, $t(30)=7.20, p<.001, d=1.32, \mathrm{BF}_{10}=$ 276145.90 , and unfamiliar-item change detection performance, $t(30)=5.27, p<.001, d=1.03, \mathrm{BF}_{10}=1959.58$.

Increasing the display time for the change detection task and changing the task order to familiarize participants with each task allowed subjects to perform better in the familiaritem change detection task than in either the 6AFC task or the unfamiliar-item change detection task; for the first time in this set of experiments, subjects were most accurate on the task for which they had the most information.

\section{Experiment 6}

The results of Experiment 5 presented two possible explanations for the improvement in the hybrid task over either individual component task. The ability to use both sources of information apparently requires either prior task experience with both tasks or a change detection task that participants can perform accurately enough to justify the effort in using it to supplement their recognition memory performance.

Experiment 6 replicated Experiment 5 but altered the task order. If performance on the familiar-item change detection suffers, we can conclude that task experience was essential for subjects to perform better in the familiar-item change detection than in either of the other tasks. If performance on the familiar-item change detection task remains superior despite the lack of experience with the other tasks, then the longer-duration, higher-accuracy change detection task presumably underlies the ability to use both sources of information.

\section{Method}

Thirty-seven undergraduate students at the University of Illinois at Urbana-Champaign participated in the experiment for course credit. The procedure was identical to that of Experiment 5 except for the task order, which reverted to that of prior experiments: (1) unfamiliar-item change detection, (2) familiar-item change detection, (3) 6AFC memory.

\section{Results and discussion}

One subject's data were excluded for falling below preregistered accuracy thresholds, leaving data from $36 \mathrm{sub}$ jects in the final analysis. Performance on the change detection task was comparable to that of Experiment $5(M=67 \%$, $S D=9 \%$ ), and performance on the 6AFC memory task was consistent with performance across all other experiments $(M=$ $66 \%, S D=18 \%$ ). As in Experiment 5, these two accuracy scores did not significantly differ from one another, $t(35)=$ $0.22, p=.83, d=0.04, \mathrm{BF}_{10}=0.18$.

As in Experiment 5, performance on the familiar-item change detection task $(M=79 \%, S D=13 \%)$ was significantly higher than both 6AFC performance, $t(35)=6.78, p<.001, d=1.24$, $\mathrm{BF}_{10}=196,011.20$, and unfamiliar-item change detection, $t(35)$ $=6.74, p<.001, d=1.20, \mathrm{BF}_{10}=176,266.20$, and the overall accuracy level was nearly identical to that of Experiment 5.

These results replicate those of Experiment 5. The longerexposure change detection task enabled subjects to increase their accuracy on the familiar-item change detection above their accuracy on the memory-only and unfamiliar change detection tasks. Altering the task order did not change the pattern of results, indicating that the altered change detection task, and not prior exposure to the two component tasks, underlies the accuracy boost.

\section{General discussion}

Our susceptibility to change blindness coexists puzzlingly with our detailed, enduring object memory. While we can retain a great deal of information over the long term, we struggle to suss out moment-to-moment changes, even dramatic ones. The results from six experiments revealed a peculiar disunity between long-term and short-term information. Subjects only seemed to use both sources of information simultaneously when they could perform each task relatively accurately, even though the information was available to them and could benefit performance even when accuracy was lower.

Experiment 1 showed that subjects do not spontaneously use familiarity information to aid in change detection, even when the presence of a familiar object perfectly indicated the location of the change. When explicitly told about the familiar objects in Experiment 2, change detection performance did 
not improve beyond the condition with completely unfamiliar objects. Although subjects could have completely ignored the change detection task, relying exclusively on recognition memory to achieve better performance, they did not do so.

In Experiments 3 and 4, subjects performed the same change detection task with familiar items, but with instructions to either use recognition memory to aid change detection or to use change information to aid recognition. In both experiments, giving subjects an explicit strategy for combining change and long-term memory information improved their performance up to the level of their 6AFC recognition accuracy, but no higher. In these studies, subjects appeared to neglect the additional information available via change detection, instead relying only long-term memory information to complete the task.

Experiments 1, 2, and 4 also explored accuracy using a 2AFC recognition memory task, providing a replication of earlier studies of memory for objects. Recognition memory in our studies was 4\%-10\% lower than previously reported $88 \%$ (Brady et al., 2008), even though our study was substantially shorter and required memory for far fewer objects. Even when subjects were required to monitor the studied stream of objects for duplicates, performance did not reach $88 \%$. This disparity could reflect differential motivation; subjects who knowingly sign up for a 5 -hour memory experiment (as in Brady et al., 2008) might be more motivated than subjects who sign up for a 1-hour experiment for course credit. While $78 \%-84 \%$ 2AFC accuracy still is impressive, object memory might not automatically be as exceptional as previously claimed and might vary across subject populations.

Experiments 1-4 showed that people do not tend to combine both long-term memory and change detection to enhance performance when tested with familiar objects. In all studies, performance would have benefitted from using both sources of information, but only in Experiments 5 and 6, when the change detection task was made easier by increasing the prechange display duration, did subjects use both.

Why might subjects disregard familiarity information when looking for a change, as they seemed to do in Experiments 1 and 2, even when they knew it could be useful? Change blindness often occurs when subjects fail to compare the current view to one held in memory (Mitroff, Simons, \& Levin, 2004; Simons, Chabris, \& Schnur, 2002). For example, $69 \%$ of subjects failed to notice the disappearance of a basketball in a real-world scenario, but when cued appropriately, $62 \%$ of those who had missed the change "discovered" memory for the ball's distinctive features (Simons et al., 2002). Drawing upon longterm memory might be more effortful than focusing on the information immediately available as part of the current task. Consistent with the idea that people minimize effort by relying on available visual information rather than drawing on long-term memory, subjects who could have performed a search task more efficiently by relying exclusively on their memory for the display's contents still relied on the visually displayed search array (Wolfe, Klempen, \& Dahlen, 2000).

In Experiments 3 and 4, rather than defaulting to using immediately available information and ignoring memory information as they seemed to do earlier, subjects used longterm information and apparently disregarded the available short-term change information. Providing a strategy illustrating the usefulness of both types of information did not induce subjects to combine it, although it did improve familiar-item change detection performance up to the level of the 6AFC recognition task.

Although subjects are disinclined to use both short-term change information and long-term memory simultaneously, we successfully coaxed them into doing so by lengthening the prechange durations in the change detection task. Thus, the failure to use both sources of information does not result from an inability to do so; tasks that demand long-term memory and tasks that rely on short-term information are not inherently competing for the same resources. Although these two sources of information are at least partially independent, subjects appear to engage in some sort of tradeoff, balancing the information gained from using a source of information against the effort necessary to use it.

When one source of information is easier to access than another, subjects might simply default to it rather than combining that information with less accessible information. For very high-effort information, such as change detection, there would be diminishing returns from using both sources; the effort required to extract the information from one source would outweigh any information value. Equate the cost-tobenefit ratio for both sources of information, however, and subjects use both.

This set of experiments indicates that when it comes to accessing potentially available and useful information, subjects do not do always; the effort required to extract the information plays a role.

\section{Limits on generality}

Based on the stability of the values we found in each of the measures across our experiments, we expect that studies using similar tasks and stimuli would replicate our pattern of results. These experiments were conducted using images of isolated objects and nonnaturalistic tasks; the pattern might not replicate with more naturalistic stimuli or with other tasks. Indeed, the lower performance on our 2AFC memory task than in earlier reports (Brady et al., 2008) indicates that accuracy might vary across subject populations. With our highly variable set of realworld object stimuli, we can be reasonably confident that the effects we observed are not limited to the particular objects in our set. 
Author note K.W. and D.J.S. jointly planned and designed the experiments. K.W. coded the experiments, conducted the analysis, and drafted the manuscript. Both authors contributed to the preregistration documentation and critically edited the manuscript. Correspondence should be addressed to Katherine Wood (kmwood2@illinois.edu).

\section{References}

Beck, M. R., Angelone, B. L., Levin, D. T., Peterson, M. S., \& Varakin, D. A. (2008). Implicit learning for probable changes in a visual change detection task. Consciousness and Cognition, 17(4), 11921208. doi:10.1016/j.concog.2008.06.011

Becker, M. W., \& Rasmussen, I. P. (2008). Guidance of attention to objects and locations by long-term memory of natural scenes. Journal of Experimental Psychology: Learning, Memory, and Cognition, 34(6), 1325-1338. doi:10.1037/a0013650

Brady, T. F., Konkle, T., Alvarez, G. A., \& Oliva, A. (2008). Visual longterm memory has a massive storage capacity for object details. Proceedings of the National Academy of Sciences of the United States of America, 105(38), 14325-14329. doi:10.1073 /pnas.0803390105

Brady, T. F., Konkle, T., Oliva, A., \& Alvarez, G. A. (2015). Detecting changes in real-world objects: The relationship between visual longterm memory and change blindness. Communicative \& Integrative Biology, 2(1), 8-11. doi:10.4161/cib.2.1.7297

Grimes, J. (1996). On the failure to detect changes in scenes across saccades. In K. Akins (Ed.), Perception (Vancouver studies in cognitive science) (Vol. 5, pp. 89-110). New York, NY: Oxford University Press.

Hollingworth, A. (2005). The Relationship Between Online Visual Representation of a Scene and Long-Term Scene Memory. Journal of Experimental Psychology: Learning, Memory, and Cognition, 31(3), 396-411. doi:10.1037/0278-7393.31.3.396

Kirby, K. N., \& Gerlanc, D. (2013). BootES: An R package for bootstrap confidence intervals on effect sizes. Behavior Research Methods, 45(4), 905-927. doi:10.3758/s13428-013-0330-5

Lakens, D. (2013). Calculating and reporting effect sizes to facilitate cumulative science: A practical primer for $t$-tests and ANOVAs. Frontiers in Psychology: Cognition, 4, 1-12. doi:10.3389 /fpsyg.2013.00863

Mitroff, S. R., Simons, D. J., \& Levin, D. T. (2004). Nothing compares 2 views: Change blindness can occur despite preserved access to the changed information. Perception \& Psychophysics, 66(8), 12681281. doi:10.3758/BF03194997
Morey, R. D., \& Rouder, J. N. (2015). BayesFactor: Computation of Bayes factors for common designs (R package Version 0.9.12-2) [Computer software]. Retrieved from https://CRAN.R-project. org/package $=$ BayesFactor

Nickerson, R. S. (1968). A note on long-term recognition memory for pictorial material. Psychonomic Science, 11(2), 2138. doi:10.3758 /BF03330991

Peirce, J. W. (2007). PsychoPy-Psychophysics software in Python. Journal of Neuroscience Methods, 162(1/2), 8-13.

R Core Team. (2015). R: A language and environment for statistical computing. Vienna, Austria: R Foundation for Statistical Computing. Retrieved from https://www.R-project.org/

Rensink, R. A., O'Regan, J. K., \& Clark, J. J. (1997). To see or not to see: The need for attention to perceive changes in scenes. Psychological Science, 8, 368-373.

Shepard, R. N. (1967). Recognition memory for words, sentences, and pictures. Journal of Verbal Learning and Verbal Behavior, 6(1), 156-163. doi:10.1016/S0022-5371(67)80067-7

Simmons, J. P., Nelson, L. D., \& Simonsohn, U. (2011). Falsepositive psychology: Undisclosed flexibility in data collection and analysis allows presenting anything as significant. Psychological Science, 22(11), 1359-1366. doi:10.1177 /0956797611417632

Simons, D. J. (1996). In sight, out of mind: When object representations fail. Psychological Science, 7(5), 301-305. doi:10.1007/s13398014-0173-7.2

Simons, D. J. \& Levin, D. T. (1998). Failure to detect changes to people during a real-world interaction. Psychonomic Bulletin \& Review 5(4), 644-649. doi:10.3758/BF03208840

Simons, D. J., Chabris, C. F., \& Schnur, T. (2002). Evidence for preserved representations in change blindness. Consciousness and Cognition, 11(1), 78-97. doi:10.1006/ccog.2001.0533

Standing, L. (1973). Learning 10,000 pictures. Quarterly Journal of Experimental Psychology, 25, 207-222.

Standing, L., Conezio, J., \& Haber, R. N. (1970). Perception and memory for pictures: Single-trial learning of 2500 visual stimuli. Psychonomic Science, 19(2), 73-74. doi:10.3758 /BF03337426

Wickham, H. (2009). Ggplot2: Elegant graphics for data analysis. New York, NY: Springer-Verlag.

Wolfe, J. M., Klempen, N., \& Dahlen, K. (2000). Postattentive vision. Journal of Experimental Psychology: Human Perception and Performance, 26(2), 693-716. doi:10.1037//00961523.26.2.693 